\title{
CIRCUIT BREAKERS TRANSIENT REDUCTION BY USING A DEDICATED CONTROLLED SWITCHING SYSTEM
}

\author{
Virginia Ivanov \\ Faculty of Electrical Engineering \\ E-mail: vivanov@elth.ucv.ro \\ Maria Brojboiu \\ University of Craiova \\ Faculty of Electrical Engineering \\ 107 Decebal Blv., 200440, Romania \\ E-mail: mbrojboiu@elth.ucv.ro
}

\author{
Sergiu Ivanov \\ Faculty of Electromechanical \\ Engineering \\ E-mail: sivanov@em.ucv.ro
}

\section{KEYWORDS}

Controlled switch, controller, circuit breaker.

\begin{abstract}
The controlled switching can be applied to any type of commutations. Now there are dedicated controllers which are used more often for the switching of the transport and distribution lines, of the small inductive loads, of the capacitors batteries, or the energizing the no load power transformers. The paper presents a microcontroller (8051 family) based system used to controlled switching of various loads. This system has two main parts: the acquiring and transfer module and the user interface, developed using the facilities specific to Graphic User Interface of Matlab ${ }^{\circledR}$.

The rate of decreasing of the dielectric stress and the mechanical dispersion of the switching on/off times qualifies the breakers to be able or not for controlled switching. The compensation of the waiting time is essential in order to obtain good performances, even for daily used breakers.
\end{abstract}

\section{THE HARDWARE ARCHITECTURE SYSTEM}

The experimental systems implies two main parts: hardware (sensors, transducers, $\mathrm{A} / \mathrm{DC}$, serial transmission) and software (field module programming, transmission protocols, user interface) (Ivanov and Ivanov 2005).

\section{Microprocessor}

The hardware part of the system is centred on the Dallas micro-controller DS87C550. This type of microcontroller is code fully compatible with the 8051 family micro-controllers, being equipped with many integrated peripherals that make it suitable for embedded applications. More than that, being equipped with the high speed core specific to the Dallas micro-controllers, the performances achieved by the hardware subsystem based on this module make it very suitable for the online monitoring of the high speed electrical equipments. The acquiring and transfer module consists of the blocks: Sources, Controller, Local console, Inputs, Outputs and Serial communication interface. By the way of three precise voltage regulators, the Sources block supplies the regulated voltages to the micro system. The Controller block is the core of the module and consists of the controller itself, the full duplex RS485 serial interface, connector to the 7 digits display, digital open collector output which can be used for commanding a power element (relay), non-volatile serial EEPROM.

The Figure 1 depicts the bloc diagram of the acquiring and transfer module.

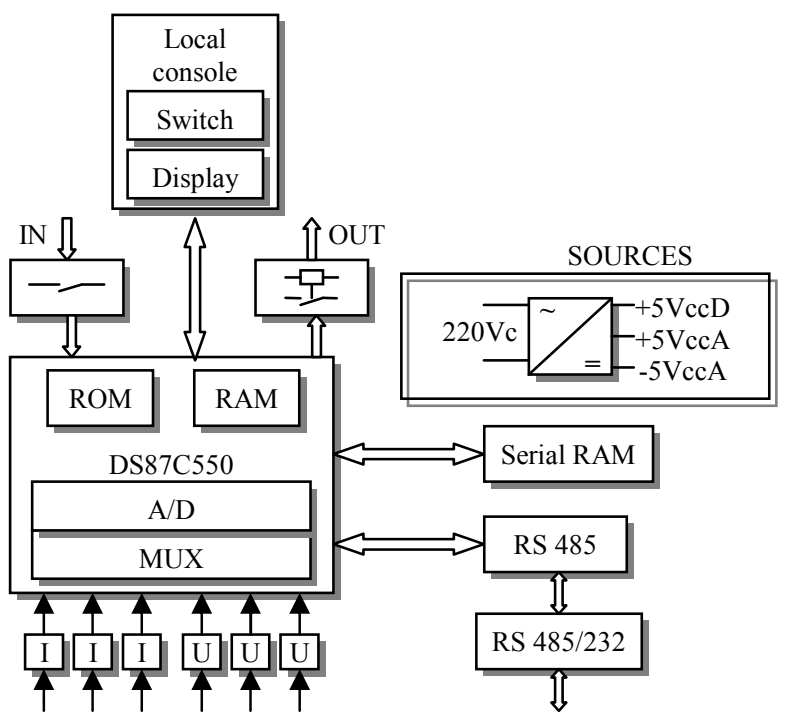

Figure 1: Main Structure of the Hardware Subsystem

\section{Measurements}

In order to integrate the mentioned component in the hardware subsystem of the monitoring structure, specific to the electrical equipments, special transducers must be used.

The system was designed to acquire all the three phase currents and voltages. As current sensors, LEM transducers LTS15-NP are used, special adapted to the integration with the micro-controller.

The voltages are measured by using three precise voltage dividers, built with $1 \%$ resistors. These dividers were designed in such manner to obtain, at the rated voltage, a trip by $\pm 0.5 \mathrm{~V}$. The $1 \%$ tolerance is quite enough, but more important is the thermal stability. For this reason, for building these dividers were used resistors with identical thermal factor. 
The compensation of the possible offset both of the current transducers and of the resistive dividers is performed a single time, at the system setting, by using the calibration routine. This task is achieved only in local-mode control, by proper configuring the micro switches. The obtained values are stored in the non volatile serial EEPROM RAM. At each start of the system, the values of the offsets are read from the serial non volatile RAM and further used for the correction of the acquired samples.

The same non volatile serial EEPROM RAM is used to store the number of the commutations performed by the installation, available at the user's demand. Due to the importance of the stored values, at start, a short routine checks that the data are not corrupted.

The domain of the possible input signals reaches $830 \mathrm{~V}$ for the voltages, 8.2A for the current respectively. We mention that in what concern the current domain, it corresponds to the $5 \mathrm{~A}$ connection of the transducers and can be easily extended to $12.3 \mathrm{~A}$ or $24.6 \mathrm{~A}$ with different connections. In order to extend the measure domains, it is also possible to have external voltage and/or current transformers.

Figure 2 depicts the hardware structure of the experimental acquiring and transfer module.

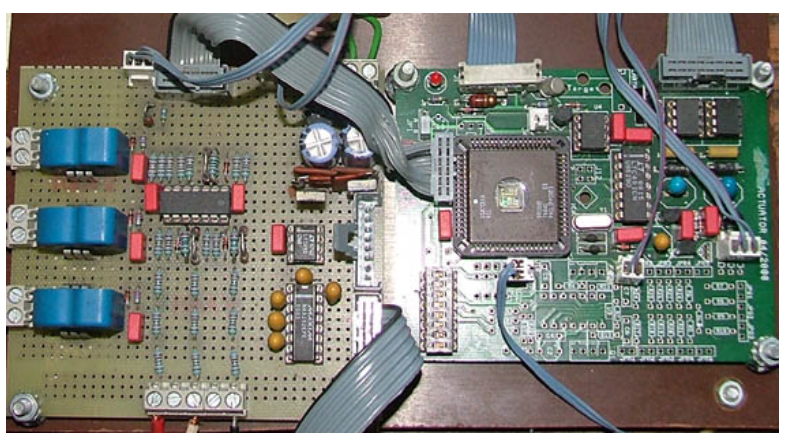

Figure 2: The Hardware Subsystem

\section{USER INTEFACE}

As medium for the development of the user interface, the Graphic User Interface (GUI) of Matlab® was chosen.

It allows the bi-directional communication with the hardware subsystem. Thus, on one hand, based on the channels chosen within the interface, it sends the proper command to the hardware subsystem which configures the proper sampling period. On the other hand, the user interface receives the sampled data and reconstitutes the signals on each sampled channel.

The interface was designed in a user-friendly manner, avoiding the programming mistakes.

As the number of acquired signals could be only one (one phase current or voltage), two (the phase current and voltage on the same phase), three (all thee phase currents or voltages), or six (all the phase currents and voltages), the interface assures the interlocking for a correct selection of the channels (Fig. 3, highlight 1).

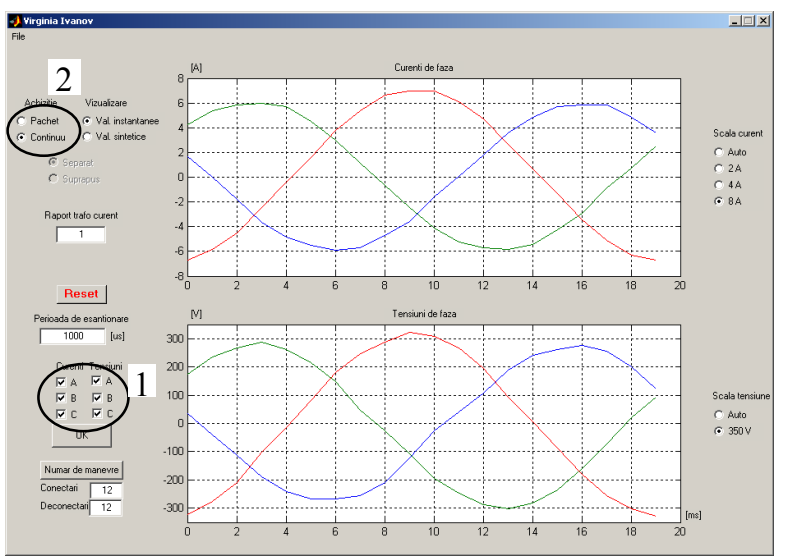

Figure 3: Interlocking of the Selected Channels

The interface allows the programming of two types of acquisitions: continuous, when the selected structure of channels is displayed continuously, or packet by packet, when each acquisition must be confirmed by the user (Fig. 3, highlight 2).

\section{PRINCIPLE OF CONTROLLED SWITCHING}

There are several important circuit breaker applications where random closing or opening instants may lead to severe voltage and current switching transients. These transients occur in the main circuits, but may also induce transients in control and auxiliary circuits, as well as in adjacent low voltage systems.

The switching transients are associated with a variety of dielectric and mechanical stresses on the high-voltage equipment, and may cause gradual or immediate damage to the system or the equipment. Induced transients may lead to a variety of disturbances, e.g. in substation control and protection systems, computers and processors, or telecommunications.

Normal energizing of shunt capacitors, shunt reactors and power transformers may cause severe transients high over-voltages, under-voltages, or high inrush currents. Upon de-energizing of shunt reactors, reignitions will occur, resulting in steep voltage surges.

The magnitude of the transients depends on the pointon-wave where closing or opening of the circuit breaker contacts occur. In a situation without controlled switching, sooner or later the switching instant will occur at the worst possible phase angle.

Controlled switching is a method for eliminating harmful transients via time controlled switching operations. Closing or opening commands to the circuit breaker are delayed in such a way that making or contact separation will occur at the optimum time instant related to the phase angle (Figure 4). 


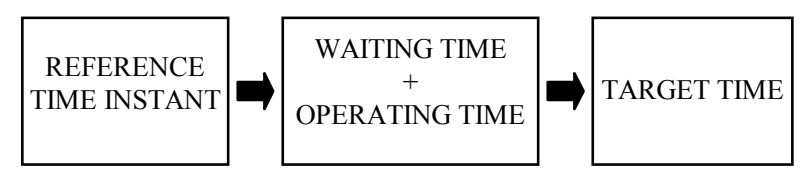

Figure 4: The Principle of the Controlled Switching

The following example illustrates the general operating principle of a controlled switching, for energizing of a capacitor bank. In order to avoid switching transients, the making instant in this case shall be at voltage zero (Catalogue publication 1HSM 2006).

For simplicity, only a single phase is considered (Figure 5), where:

$\mathrm{T}_{\mathrm{F}}=$ Time to detect final reference voltage zero;

$\mathrm{T}_{\mathrm{V}}=$ Waiting time;

$\mathrm{T}_{\mathrm{M}}=$ Expected make time of circuit breaker.

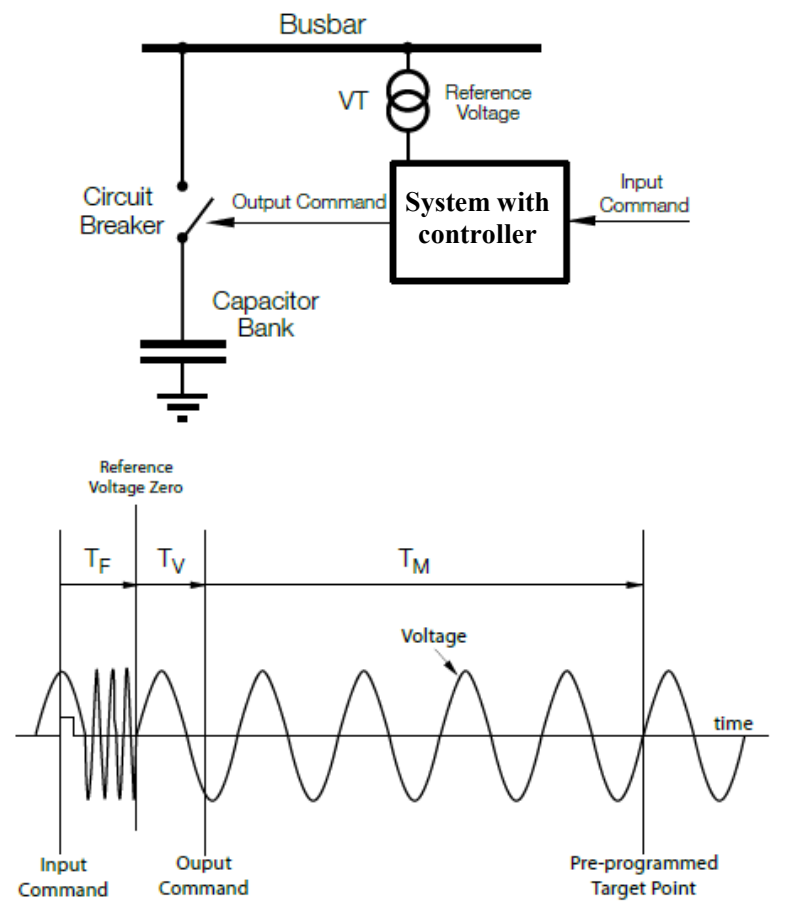

Figure 5: Principle of a Controlled Switching for Energizing a Capacitor Bank

\section{TESTING PROCEDURES AND CONDITIONS FOR SINGLE COMPONENTS AND INTEGRATED SYSTEMS}

The basic conditions imposed to independent poles breakers refer to (CIGRE WG13.07 2001):

1. The negative slope (rate of decreasing) of the dielectric stress (RDDS);

2. The mechanical dispersion of the switching on / off times, depending on various conditions;

3. The dependence between the pause time and operation time.

The rate of decreasing of the dielectric rigidity and the mechanical dispersion of the switching on/off times qualifies the breakers to be able or not for controlled switching and give an optimum for different switching applications. The compensation of the waiting time is essential in order to obtain good performances, even for daily used breakers.

The controller must be able to implement the folowing functions (Avent 2002):

1. compensation of the waiting time face to the increasing of the closing time due to the pause time;

2. conditional compensation of the turning on / off times depending on various conditions (ambient temperature, command voltage, hydraulic pressure);

3. adaptive compensation of the changes of the turning on / off times due to the long term ageing.

The influence of the pause time on the driving mechanism is one of the mainsprings of the command errors. Till recent, the most controllers did not compensate the pause time.

Figure 6 shows the dependence of the closing time versus the pause time for the spring mechanism specific to $145 \mathrm{kV}-362 \mathrm{kV}$ systems. Figure 7 plots the same dependency for hydraulic mechanisms specific to $300 \mathrm{kV}$ systems (Ito 2002).

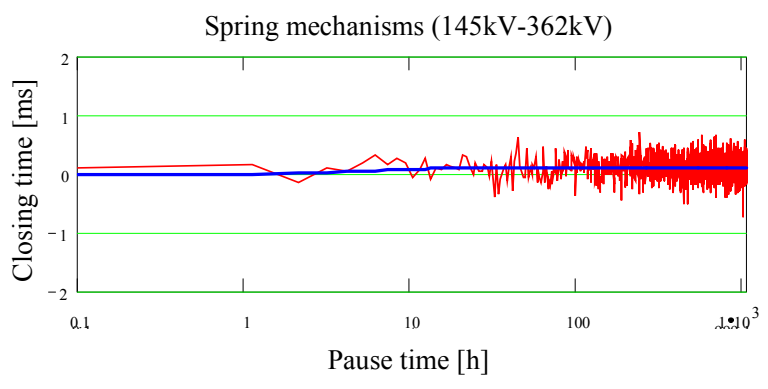

Figure 6: Closing Time for Spring Mechanisms

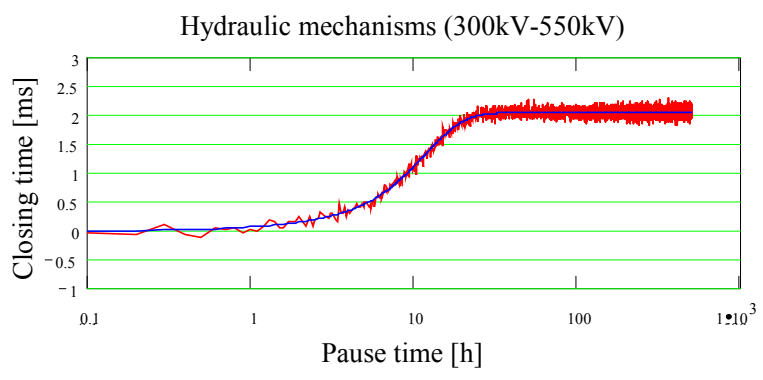

Figure 7: Closing Time for Hydraulic Mechanisms

These characteristics were evaluated for cycles C-O-C$\mathrm{O}$ after pause times of 2, 4, 8, 16, 64, 128, 256, 720 hours. The spring mechanism was greased on the moving parts. It has a very low dependency of the closing time by the pause time, up to 1000 hours. The experiments with a classic hydraulic mechanism show the increasing of operation (closing) time can be noticed for pause time of few hours. For pause time greater than $70 \mathrm{~h}$, the closing time increases by $2 \mathrm{~ms}$. The pause time must be continuously compensated for pause times up to $100 \mathrm{~h}$. 
The most controllers have conditional compensations which are able to adjust the waiting time following the changes of the opening/closing times due to the ambient temperature, command voltage or hydraulic pressure. The variations of the turning on / off times of a $145 \mathrm{kV}$ independent poles gas breaker (spring mechanism) by the control voltage and ambient temperature were studied. The dependency of the closing / opening time on the control voltage and temperature were measured for 40 manoeuvres. Figure 8 plots the deviations of the closing / opening time by the average values for different ambient temperatures (Tsudata 2002).
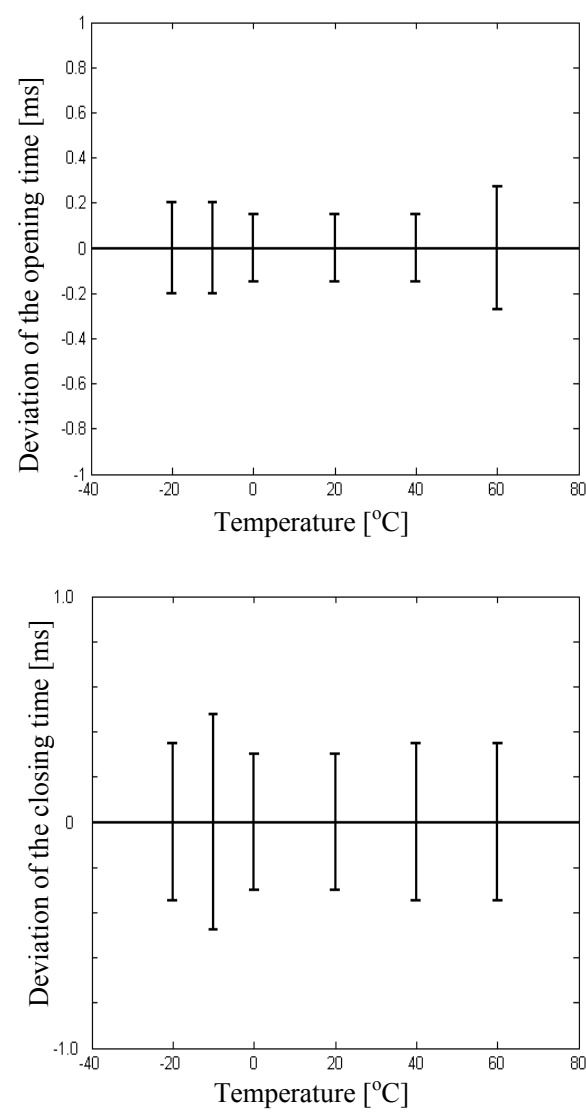

Figure 8: Closing / Opening Time for Different Temperatures

The figures show that, by using the controlled switching, the error on the desired opening / closing time can be reduced below $1 \mathrm{~ms}$. The differences by the typical switching time can be represented as a surface. This surface can be stored in the controller's memory as a common characteristic of a specific breaker type. This is possible because the average opening / closing time have low variations due to the manufacturing dispersions.

The gas breakers generally imply, during opening / closing operations, sliding parts (contacts, gaskets). Consequently, the operation characteristics are strongly influenced by the changes of the friction forces due to the ageing and fraying. As the changes are slow, the adaptive control can effectively compensate the deviations of the operation times due to multiple manoeuvres. The effects of the adaptive control depend on the number of manoeuvres previous supervised and by the weight factors. These parameters are determined following a deep research of mechanical endurance tests.

Figure 9 shows the typical deviations of the closing time, measured without and with adaptive control, for 1500 manoeuvres of a $145 \mathrm{kV}$ gas breaker (spring mechanism) (Ito 2002). The variation of the closing time is given as the difference between the estimated closing time and the effective one. Even the closing time is greater when the number of manoeuvres increases, this influence can be effectively be compensated quite precisely by the mean of the adaptive control. If the controller is able to detect the closing instant by measurement of the current in the principal circuit, the rate of decreasing of the dielectric stress can be also be compensated by adaptive control.
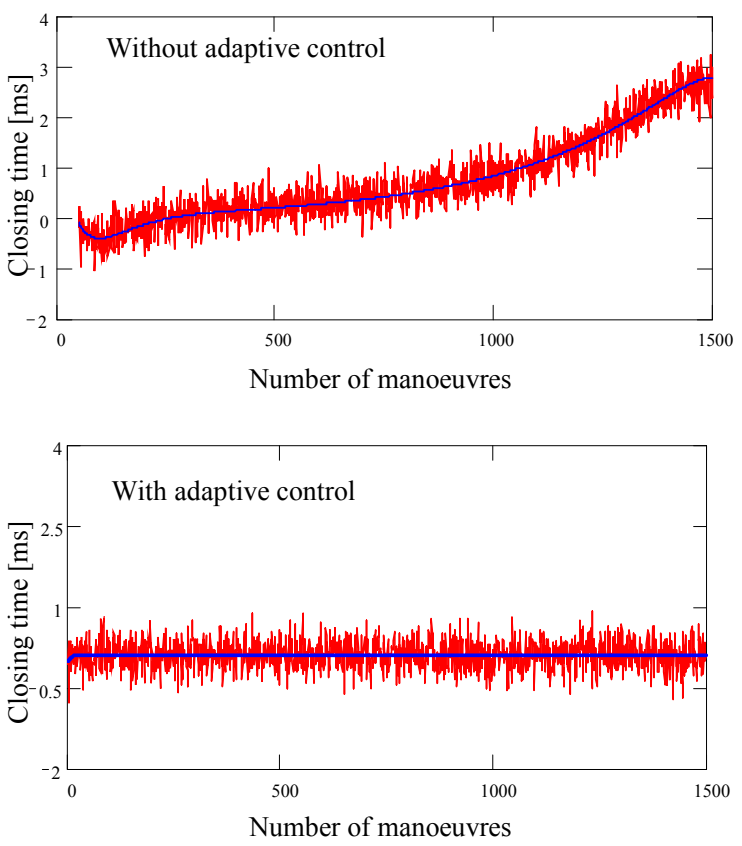

Figure 9: Closing Time with and without Adaptive Control

\section{CONCLUSIONS}

In order to reduce the stress of the switching equipment, the controlled commutation can successfully be applied, for the most applications.

The controlled switching systems request a precise understanding of the switching phenomenon. The paper highlights the importance of the compensations of the operation times. Some errors may be generated by the deviation of the operation time due to an insufficient compensation, especially du to the pause time. Other factors which influence the closing / opening times and which must be compensated are the ambient temperature, command voltage or hydraulic pressure. 
The microcontroller system described in the paper can compensate the closing / opening times and eliminates the control errors, for different types of loads, depending on the specific characteristics of the breaker's circuit and on the commutations conditions.

\section{REFERENCES}

Avent, B. L. 2002. "Application of 500kV Circuit Breakers on Transmission Line with MOV Protected Series Capacitor Bank." Communications of the 2002 CIGRE Session Paper, 13-107.

Catalogue publication 1HSM 9543 22-01 en. 2006. Controlled Switching, Buyer's Guide, Application Guide. Edition 2. 2006-09, http://www.abb.com.

Catalogue publication CIGRE WG13.07 2001. Controlled Switching of HVAC Circuit Breakers: Planning, Specification and Testing of controlled switching systems. In ELECTRA No. 197 2001, 23-33.

Ito, H. 2002. "Controlled Switching Technologies". In Stateof-the-Art. IEEE, 1455-1460.

Ivanov, V. and S. Ivanov. 2005. "High-Speed Monitoring System for Electromechanical Equipments". In Proceedings of the 2005 International Symposium on Advanced Electromechanical Motion Systems (Lausanne, Switzerland).

Tsudata, H. 2002. "Controlled Switching System for Capacitor Bank and Transformer Switching". In Electrical Engineering. 2125-2130.

\section{AUTHOR BIOGRAPHIE}

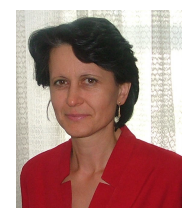

VIRGINIA IVANOV was born in Vela, Dolj, Romania, 1963. She was graduated in Electrical Engineering at University of Craiova, Romania, in 1986 and Doctor in Electrical Engineering in 2004. From 1986 to 1998 she worked as researcher with the Researching Institute for Motors, Transformers and Electric Equipment Craiova. In 1998 she joined the Faculty for Electrical Engineering, Department of Electrical Equipment and technologies.

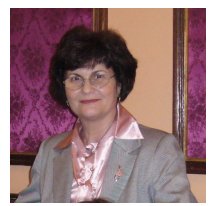

MARIA D. BROJBOIU was born in 1952 in the town Pucioasa Romania. She is currently working as Professor at the University of Craiova, Electrical Engineering Faculty, Department of Electrical Equipment and Technologies since 1981. Before that, she worked as design engineer at the Electroputere holding the Research and Development Center. She is Doctor in Science Technique - Electrical Engineering.

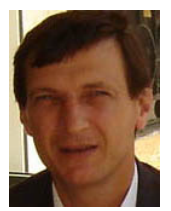

SERGIU IVANOV was born in Hunedoara, Romania and went to the University of Craiova, where he studied electrical engineering and obtained his degree in 1986. He worked for the Institute for Research in Motors, Transformers and Electrical Equipment Craiova before moving in 1991 to the University of Craiova. He obtained his $\mathrm{PhD}$ in 1998 with a topic in the field of the control of the electric drives systems. He is involved in modelling of the electromechanical systems. 